\title{
Optimising calcium monitoring post thyroid and parathyroid surgery
}

Eleanor Rayner, Nerida Williams, Julie Dunn

Royal Devon \& Exeter NHS Foundation Trust, UK

\begin{abstract}
There is a risk of hypocalcaemia following total thyroidectomy and parathyroidectomy surgery, with the nadir being two to five days post operatively.[1] This project examined and improved the post-operative monitoring of serum calcium in patients undergoing these procedures at the Royal Devon and Exeter (RD\&E) Hospital over a ten month period in 2013. A retrospective audit was undertaken, identifying whether serum calcium was monitored according to guideline; ie measured within the first 24 hours of surgery and daily thereafter until the patient was normocalcaemic.[2-3] The audit showed an overall compliance in $79 \%(n=34)$ of cases. Of the non-compliant cases $(n=9)$, seven also suffered hypocalcaemia.
\end{abstract}

The current process was mapped by a flow chart and used as a basis for discussing experiences and identifying areas for improvement. Interventions implemented included a patient leaflet, a process flow chart displayed in the team office and in the weekend handover book, standard text for use in discharge summaries describing the process to GPs, the issuing of prescriptions or actual supplements for patients felt to be at high risk of hypocalcaemia, and finally education to the wider surgical junior doctor team. Percentage compliance with guidelines was compared before and after intervention with a re-audit undertaken in April 2014. Significant improvement was shown, with $100 \%$ of re-audit cases compliant $(n=41)$, and all seven cases of hypocalcaemia were managed in full compliance with guideline.

\section{Problem}

There is a risk of hypocalcaemia following total thyroid and parathyroid surgery, with the nadir being two to five days post operatively.[1] At the Royal Devon \& Exeter Hospital, UK, patients are discharged on the day after surgery so calcium testing and management is done as an outpatient during this critical time. The process was felt to be non-robust with ad-hoc arrangements being made for each patient. To add further complication to this issue, the majority of these operations are performed on a Wednesday, meaning patients at risk of hypocalcaemia often require monitoring over the weekend.

Regular team members were frequently not present at the weekend, so there was a reliance on handover to on-call staff at the most critical period. Key testing windows were often missed and weekend staff did not feel confident with the process. Patients were unsure of what they would need to do and what the symptoms of hypocalcaemia would be. In some cases it had been logistically difficult to get supplements to patients who became hypocalcaemic over the weekend, relying on out of hours general practitioners (GPs) to issue the required prescription. In addition to all this, GPs who were unsure of the process had also been giving conflicting advice to patients on whether they needed further testing or calcium supplementation.

\section{Background}

Both total thyroidectomy and parathyroidectomy procedures carry the risk of post-operative hypocalcemia. Between January and September 2013 the breast and endocrine surgery unit at the Royal Devon and Exeter Hospital performed 23 total thyroidectomies, 21 thyroid lobectomies (of which two were completions), and 15 parathyroidectomies.

Hypocalcaemia can result in patients suffering a variety of signs and symptoms, the severity of which usually correlates with the magnitude and rate of the fall.[4] In light of this, thyroidectomy and parathyroidectomy patients are at greater risk of becoming symptomatic as they may have been previously hypercalcaemic for several months and calcium levels may begin to decline immediately after surgery and continue to do so for several days before stabilising. Common symptoms patients may experience include paraesthesia and tingling, particularly around the mouth and in the fingers and toes, and also muscle spasms and cramps. Lower levels of calcium are associated with more serious signs and can result in seizures, bronchospasm, laryngospasm, and a prolonged QT interval which may progress to cardiac arrhythmia's such as ventricular fibrillation or heart block.[4]

As both total thyroidectomy and parathyroidectomy procedures carry this risk, The Royal College of Physicians and British Thyroid Association guideline states that "serum calcium should be checked on the day after surgery and daily until the hypocalcaemia improves. A decline in serum calcium concentration in the 24 hours after surgery is predictive of the need for calcium supplementation."[2,3,5]

\section{Baseline measurement}

A retrospective audit examined the post operative monitoring of all patients who underwent a total thyroidectomy or parathyroidectomy over a nine month period in 2013. We identified whether serum calcium was monitored according to the guideline; ie measured within the first 24 hours of surgery and daily thereafter until the 
patient was normocalcaemic.[2-3] Data were collected from the hospital pathology system on the timing, number of tests, and the values of the patients calcium level postoperatively.

Forty-three procedures were identified and the audit showed an overall compliance in $79 \%(n=34)$ of cases. Of the non-compliant cases $(n=9)$, seven patients also suffered hypocalcaemia.

See supplementary file: ds3816.png - "Figure 1- Initial Calcium Monitoring Process"

\section{Design}

The current process was mapped by a flow chart (figure 1). The junior doctors and consultant surgeon used this flow chart as a basis for discussing their experiences and identifying areas within the patient journey that could be improved.

Five main strategies of improvement were decided:

1. A patient information handout was designed that could be provided on discharge. This contained the symptoms of hypocalcaemia and the telephone numbers to contact for assistance, both in and out of hours (figure 2). This same handout also doubled as an area to record dates and times of blood test appointments and the patients calcium result for monitoring trends

2. Standard written text was created that would be included in all total thyroidectomy and parathyroidectomy discharge summaries. This explained to the GP the risk of potential hypocalcaemia post-operatively and explained that the discharging surgical team were managing this issue and liaising with the patient directly regarding further measurement and any necessary management (figure 3 )

3. A flow process chart was displayed in the surgical team office and in the weekend handover book to simplify and unify calcium monitoring (figure 4)

4. Where it was felt by the consultant surgeon that patients were at a high risk of developing hypocalcamia, actual prescriptions or calcium supplements were provided on discharge with instructions that we would be in contact to discuss dosing and regime if required

5. Finally, education via email and face to face discussions for the wider surgical junior doctor team was undertaken regarding how to use the process over the weekend and the importance of getting it right.

\section{Strategy}

The junior doctors who identified the problem were the key people responsible for carrying out the changes and they involved the wider junior doctor team who would be responsible for the process over weekends. Proposed changes to the process were mapped out and supporting documentation drafted. The process change was implemented over a one month period starting in October 2013 and targets of $>95 \%$ compliance plus zero incidences of noncompliance in patients suffering hypocalcaemia were set. All five interventions were initially implemented at the same time, however some strategies necessitated that several PDSA cycles be completed during this time in order to gain the desired results and achieve patient and doctor satisfaction.

\section{- Patient information leaflet}

PDSA cycle 1: The initial patient information leaflet was designed to remind patients of the symptoms of hypocalcaemia and to provide them with the telephone numbers to contact for assistance if required. This was received well by patients, reducing their anxiety at the point of discharge. However, doctors were providing them with separate pieces of paper detailing their next blood test appointment in addition to this leaflet. This felt excessive and that the two should be combined.

PDSA cycle 2: A new information leaflet was designed that also contained space to record the date, time, and location of the follow up monitoring. Additionally it highlighted that it would also be helpful to have the patient's recent calcium level to hand when answering questions and discussing testing. The new leaflet was therefore edited to include space for patients to record their most recent calcium level next to the corresponding appointment space. This also allowed doctors to see trends without needing to log onto the hospital computer system.

PDSA cycle 3: The new information leaflet was distributed on the morning of discharge to all patients undergoing a total thyroidectomy or parathyroidectomy. This received positive feedback from the patients, junior doctors, and consultant; so much so it was felt that producing a similar "patient record card" for use only by medical staff could be beneficial, particularly for on-call staff required to monitor patients over the weekend.

PDSA cycle 4: A corresponding record card designed to be kept on site was produced that contained space for recording the time, date and location of future monitoring appointments as well as the patients calcium result. This card also included contact details for the consultant who was to be contacted regarding clinical decisions. This card was designed to be passed to the doctor responsible for chasing the patient's calcium result and then filed in the medical notes when testing had ceased. Breast and endocrine team juniors implemented this form and passed it to the junior doctor on-call responsible for the monitoring out of hours. Many juniors were keen to have more information regarding the patient and their procedure and therefore an additional section for "notes" was included within the card.

PDSA cycle 5: Final medical record cards were produced that provided space for additional information to be documented if required. The use of the two cards once implemented provided greater patient and doctor satisfaction and reduced the possibility of missed appointments (figure 2).

\section{- Standard text for discharge summaries:}

PDSA cycle 1: We had previously highlighted that conflicting information had sometimes been given to patients regarding 
monitoring and managing of their calcium level due to poor communication between GP's and the surgical team. In light of this, standard written text was produced and included in all total thyroidectomy and parathyroidectomy discharge summaries that explained the risk of potential hypocalcaemia post-operatively, and that the discharging surgical team are managing this issue and liaising with the patient directly regarding further measurement and any necessary management (figure 3). This was implemented by the junior doctors within the breast and endocrine team, although it was noted that issues arose when juniors were required to write summaries on computers away from their usual wards as the standard text was not available.

PDSA cycle 2: Standard text for inclusion in discharge summaries was added to the common surgical drive and was therefore available on all trust computers.

\section{- Calcium monitoring process flow-chart}

PDSA cycle 1: It was felt that a flow chart to act as a prompt for all team members would be beneficial in ensuring that the guideline was followed and patients were monitored appropriately. This was designed and displayed in the breast and endocrine surgical team office. All members of the team found this useful and after discussion with the wider junior doctor staff, who were less familiar with the process but required to be responsible for the monitoring out of normal working hours, it was felt the chart should be available to them also.

PDSA cycle 2: A copy of the flow diagram that detailed the monitoring process was placed in the doctors handover book that is used by all on-call junior staff. This was highly effective, simplifying and unifying calcium monitoring post-operatively (figure 4).

\section{- Precautionary prescriptions of calcium supplements}

Precautionary prescriptions or calcium supplements were provided on discharge by the surgical team where it was felt by the consultant surgeon that certain patients were at a high risk of developing hypocalcaemia, with instructions that we would be in contact to discuss dosing and regime if required. This was aimed at reducing the need to involve out of hours community services (such as Devon Doctors or the walk-in-centre) if a patient was subsequently found to be hypocalcaemic and requiring calcium supplementation over the weekend.

\section{- Education of junior staff}

Education via email and face to face discussions for the wider surgical junior doctor team was undertaken regarding how to use the process over the weekend and the importance of getting it right. This education also included demonstration of how to use the patient record cards and where to put them once monitoring had ceased.

\section{Results}

A re-audit was carried out using the same parameters as the initial audit in April 2014, allowing for six months of procedures to be included to ensure comparable numbers. Percentage compliance with guidelines was compared before and after the five interventions. Significant improvement was shown, with $100 \%$ of reaudit cases compliant $(n=41)$ compared to the initial audit showing only $79 \%$ compliance $(n=34)$ of the 43 cases. Re-audit also demonstrated that after the intervention within the further 41 operations, seven instances of hypocalcaemia had been recorded. However, all of these cases had been managed in full compliance with the guideline in comparison to none within the initial audit cycle.

See supplementary file: ds3764.png - "Calcium Monitoring Improvement Strategies"

\section{Lessons and limitations}

The changes resulted in more confident and robust monitoring of calcium by the junior doctors. Patients knew where and when to get their calcium tested and the signs of low calcium to look out for. They had a direct means of getting in touch with the team during working hours and the on-call team out of hours. The timely testing and checking of results enabled the consultant to make decisions on management with the best available data. GPs were kept up to date with the process. Junior doctor and consultant satisfaction improved as the system felt more streamlined with increased patient safety and care. Patient satisfaction also increased and in particular the use of precautionary prescriptions was received especially well. These helped to reduce patient anxiety around the possibility of developing hypocalcaemia.

Getting early buy in from the leadership team, using individual face to face communication with staff members and patients, and backing this up with simple yet effective documentation were the key elements to the project's success. Sample size of this project was limited by the number of operations being performed and overall to ensure large enough groups for comparison before and after our interventions, sixteen months worth of procedures were included in total.

If we repeated the project we would have liked to have gained qualitative data from both patients and staff on their opinion of the current process prior to the implementing changes and repeated this survey afterwards to further objectively assess the success of the project. To ensure sustainability, current team members are required to provide a formal induction and handover regarding the post-operative calcium monitoring process to the new junior doctors on the firm prior to moving rotations to help ensure that standards are maintained.

\section{Conclusion}

Ensuring that patients are monitored appropriately and supplementation provided where necessary is vital to maintain patient safety at all times due to the significant consequences that are associated with a rapidly falling calcium level. Mapping the current process by flow-chart and using this as the basis for 


\section{BMJ Quality Improvement Reports}

discussing experiences and identifying areas for improvement was a crucial first step towards achieving this. Implementing five simple interventions has been shown to greatly improve the monitoring of calcium post-operatively, and ultimately improve patient safety at the RD\&E.

\section{References}

1. Bentrem DJ, Rademaker A, Angelos P. Evaluation of serum calcium levels in predicting hypoparathyroidism after total/near-total thyroidectomy or parathyroidectomy. Am Surg 2001;67:249-51.

2. British Thyroid Association, Royal College of Physicians. Guidelines for the management of thyroid cancer (Perros $P$, ed) 3rd edition, p29: Early post-surgical management. Clinical Endocrinology. Report of the Thyroid Cancer Guidelines Update Group. London: Royal College of Physicians, Revised July 2014.

3. Szubin L, Kacker A, Kakani R, Komisar A, Blaugrund S. The management of post-thyroidectomy hypocalcemia. Ear Nose Throat J 1996;75:612-4,616.

4. Patient.co.uk. Patient Plus Article: Hypocalcaemia. Last updated 2011. Accessed 10th April 2014. Available from: http://www.patient.co.uk/doctor/Hypocalcaemia.htm

5. Oltmann SC1, Brekke AV, Schneider DF, Schaefer SC, Chen H, Sippel RS. Preventing Postoperative Hypocalcemia in Patients with Graves Disease: A Prospective Study. Ann Surg Oncol 2014 [Epub ahead of print].

\section{Declaration of interests}

Nothing to declare. 